REGARDS

SUR LECONOMIE ALLEMANDE

BULLETIN ECONOMIQUE DU CIRAC
Regards sur l'économie allemande

Bulletin économique du CIRAC

$70 \mid 2005$

Varia

\title{
Démographie et compétitivité
}

\section{Stefanie Wahl}

Traducteur : Isabelle Bourgeois

\section{OpenEdition}

\section{Journals}

Édition électronique

URL : http://journals.openedition.org/rea/1903

DOI : 10.4000/rea.1903

ISBN : 978-2-8218-0836-2

ISSN : 1965-0787

Éditeur

CIRAC

Édition imprimée

Date de publication : 1 mars 2005

ISSN : 1156-8992

Référence électronique

Stefanie Wahl, « Démographie et compétitivité », Regards sur l'économie allemande [En ligne], 70 | mars 2005, mis en ligne le 05 novembre 2009, consulté le 01 mai 2019. URL : http://

journals.openedition.org/rea/1903; DOI : 10.4000/rea.1903 


\title{
Démographie et compétitivité
}

\author{
Stefanie Wahl
}

Lors du sommet de Lisbonne en mars 2000, les chefs d'Etat et de gouvernement européens s'étaient fixé un objectif ambitieux à l'horizon 2010: faire de I'UE " l'économie de la connaissance la plus compétitive et la plus dynamique " du monde. A l'origine de ce qu'il est convenu d'appeler la "stratégie de Lisbonne ", il y avait un constat: l'écart se creuse entre la dynamique de croissance de l'UE et celle de l'Amérique du Nord comme de l'Asie. L'Europe doit en effet affronter le double défi d'une population qui ne croît plus que faiblement et, surtout, qui vieillit.

D'ores et déjà, il faut reconnaître que l'objectif de Lisbonne ne pourra pas être atteint; et ce, pour de multiples raisons. L'une d'entre elles, et non des moindres, réside dans l'évolution démographique : le vieillissement inexorable de la population européenne bride la dynamique de croissance de l'UE, notamment en générant des valeurs et des modes de comportement inconciliables avec l'objectif ambitieux de Lisbonne. L'accélération attendue du vieillissement après 2010 contribuera en outre à tasser la compétitivité européenne pour de longues années. On peut se consoler à l'idée que d'autres régions du monde, comme la Chine ou l'Asie du sud-est, sont confrontées elles aussi au vieillissement démographique, bien qu'avec un certain décalage dans le temps. Mais une fois de plus, l'Europe joue un rôle de précurseur dans une évolution mondiale, ce qui fait de la maîtrise du problème démographique une priorité absolue pour l'UE. Les Etats membres devraient dès lors se préparer plus systématiquement que par le passé à affronter le défi d'une population en recul et de plus en plus âgée.

\section{Le défi démographique}

L'Europe, plus précisément l'UE, subit déjà une profonde mutation démographique. Certes, la population européenne a augmenté de 70 millions de personnes entre 1970 et 2005 pour passer de 656 millions à 726 millions. Mais dans le même temps, elle a beaucoup vieilli : au cours des 35 dernières années, l'âge médian est passé de 32 à 39 ans. Et alors que la part des moins de 20 ans s'est réduite d'un gros tiers à un petit quart, celle des plus de 59 ans est passée d'un bon sixième à un cinquième de la population totale. Les chiffres absolus sont encore plus parlants : le nombre des Européens de plus de 59 ans s'est accru de 49 millions ; celui des plus de 79 ans a doublé, passant de près de 11 à désormais 21 millions. Dans le même temps, le nombre des moins de 20 ans reculait de 56 millions. Le pays le plus fortement touché par cette évolution est l'Allemagne : un quart de la population a aujourd'hui plus de 59 ans (un cinquième en 1970), et seulement un bon cinquième moins de 20 ans (contre $30 \%$ auparavant). L'âge médian est passé en conséquence de 34 ans à 42 ans.

Ce vieillissement a pour cause la conjonction de deux tendances : d'un côté des taux de natalité en faible croissance, voire en recul ; de l'autre, une hausse de l'espérance de vie. Alors qu'en 1970, une Européenne donnait naissance à 2,3 enfants en moyenne, ce qui permettait d'assurer le renouvellement de la population, elle ne met plus au monde aujourd'hui que 1,4 enfant. Aujourd'hui donc ne naissent que les deux tiers du nombre d'enfants qui serait nécessaire

L'âge médian des Européens est aujourd'hui de 39 ans

1,4 enfant par femme et une espérance de vie de 74 ans 
En 2050, plus d'un tiers des Européens aura plus de 59 ans pour assurer le maintien du niveau de population. S'ajoute à cela qu'en 1970 , l'espérance de vie à la naissance atteignait en moyenne un peu plus de 71 ans ; elle est aujourd'hui de 74 ans. Certes, les disparités régionales sont fortes au sein de l'Europe : les PECO enregistrent les plus faibles taux de natalité, les pays ouest-européens ayant l'espérance de vie la plus élevée. Mais il n'en reste pas moins que toute l'Europe est confrontée au vieillissement démographique et, à plus ou moins brève échéance, au recul de sa population.

Si, conformément aux prévisions, le taux de natalité reste inchangé d'ici 2050, que l'espérance de vie progresse à 81 ans en moyenne et que quelque 52 millions d'étrangers (chiffres nets) immigrent en Europe, la population européenne comptera à terme 597 millions de personnes - soit 129 millions de moins qu'actuellement. Un large tiers de ces personnes aura alors plus de 59 ans, et un sixième seulement, moins de 20 ans. La structure de la pyramide des âges se sera alors inversée par rapport à 1970. Pour le dire autrement : le nombre des plus de 59 ans sera supérieur de 72 millions à son niveau actuel ; pour moitié, cet accroissement sera imputable aux plus de 79 ans.
Evolution de la population européenne 1970 - 2050

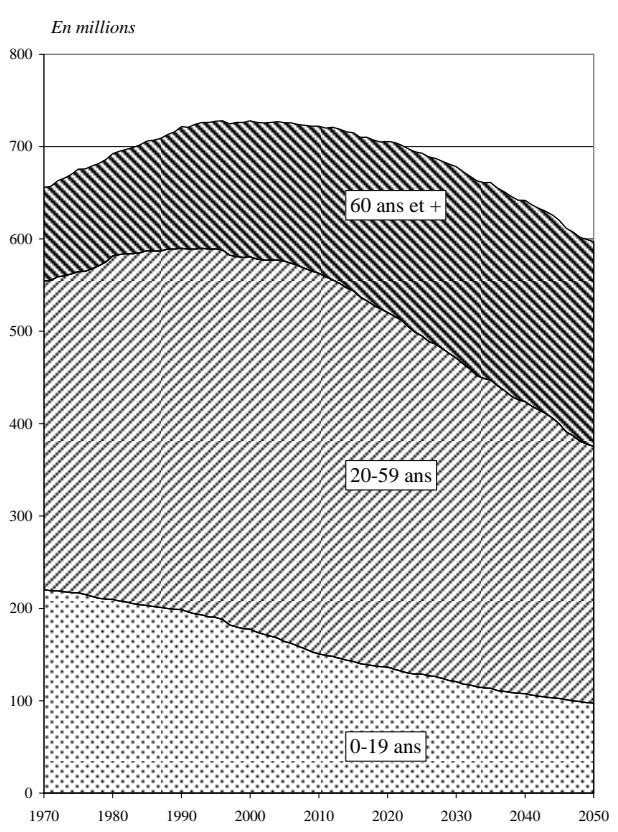

Source : OCDE
Evolution de la population allemande 1900 - 2050

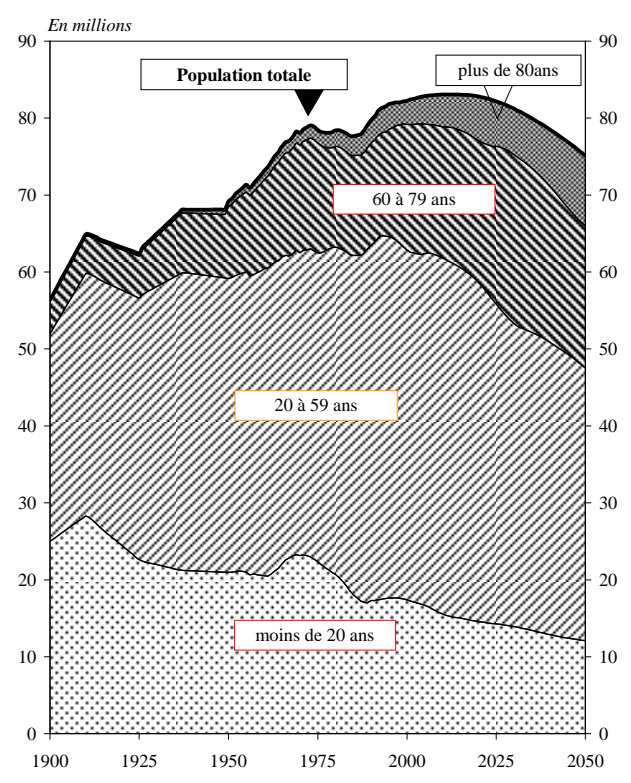

Source: Statistisches Bundesamt, 10. koordinierte Bevölkerungsvorausbe rechnung mit jährlicher Nettozuwanderung von 200.000, et IWG BONN
L'âge médian des Européens sera alors de 48 ans
Avec un âge médian de 48 ans en 2050, l'Europe sera alors bel et bien un « vieux continent ». Les Américains, en comparaison, seront alors presque aussi jeunes que les Européens d'aujourd'hui : leur âge médian sera alors de 40 ans, la part des personnes âgées dans la population s'élèvera à $26 \%$ seulement - soit le taux qu'atteindra l'Europe dès 2020. Bien que le vieillissement démographique les affecte eux aussi, les Indiens seront plus jeunes que les Américains, avec un âge médian de 38 ans. Quant aux Chinois, à l'inverse, leur âge médian se situera à 44 ans en 2050 ; c'est là l'effet de la sévère politique de limitation des naissances à 1 enfant par foyer. Certes, avec 30 \%, la part des plus de 59 ans dans la population chinoise restera inférieure à la nôtre, mais en l'espace de 45 ans, elle aura triplé, traduisant l'accélération du vieillissement démographique - un phénomène qui va poser à la Chine des problèmes quasiinsolubles dans les décennies à venir. 


\section{Les conséquences du vieillissement démographique}

La tendance au recul de la population européenne et, plus encore, la modification durable de la structure de sa pyramide des âges, auront des conséquences dramatiques qui affecteront l'économie et la société dans leur ensemble. Car elles ne seront pas seulement d'ordre objectif, mais aussi de nature subjective étant donné qu'elles influeront sur les comportements et les valeurs des Européens. Dans un premier temps, les Européens devront concentrer leurs efforts sur la gestion de ces mutations démographiques, ce qui monopolisera leur énergie. En conséquence, l'Europe verra se réduire son influence comme sa compétitivité internationales. Mais si à moyen terme, et même sur le long terme, elle parvient à s'adapter à la nouvelle donne démographique, sa population sera mieux armée pour l'avenir que nombre de ses concurrents non européens qui, eux, n'en seront alors qu'au stade où ils commenceront à affronter les mutations induites par le vieillissement.

Arithmétiquement, dans un contexte mondial de croissance démographique, l'Europe comptera moins. Alors qu'aujourd'hui, les Européens représentent $11 \%$ de la population mondiale, ils ne seront plus que $7 \%$ en 2050 . Le poids économique de l'Europe diminuera en conséquence, sous le double effet des mutations démographiques et de la montée en puissance des économies émergentes chinoise et indienne. Or l'ampleur de sa perte de compétitivité mondiale dépend essentiellement de la capacité de l'Europe à adapter ses structures et institutions à une population vieillissante et en recul, comme à compenser les déficits et handicaps liés à cette évolution.

Celle-ci affectera directement le potentiel de main d'œuvre : en 2050, la population européenne en âge de travailler (les personnes de 15 à 64 ans) aura reculé de 146 millions par rapport à son niveau actuel, soit une diminution de $29 \%$. Même si tous les Etats membres allongent la durée de la vie professionnelle, incitent massivement les femmes à entrer dans la vie active et réduisent le chômage, le nombre des actifs sera toujours inférieur en 2050 à ce qu'il est actuellement. Or dans le même temps, ceux-ci devront subvenir aux besoins d'un nombre nettement accru de personnes ayant cessé leur activité. Alors qu'aujourd'hui, on compte 23 personnes de plus de 65 ans pour 100 Européens âgés de 15 à 64 ans, ce nombre aura plus que doublé en 2050. Mais ces moyennes masquent de fortes disparités au sein de l'UE. Si par exemple, 100 Danois en âge de travailler devront subvenir aux besoins de 36 retraités en 2050, les Italiens auront à charge, eux, 61 personnes de plus de 65 ans - soit un retraité par actif occupé.

Selon diverses prévisions, sans modification des systèmes européens de protection sociale, le vieillissement démographique se soldera d'ici 2050 par une hausse de près de 8 points de pourcentage du PIB des seules dépenses de santé et de protection vieillesse. La solidarité générationnelle constituera alors une charge très lourde pour les futurs actifs.

Pour compenser la diminution de la population active, les Européens en âge de travailler devront accroître leur productivité. Or ils vieillissent eux aussi. En Allemagne par exemple, l'âge moyen des actifs occupés passera d'un peu moins de 40 ans actuellement à 43,5 ans en 2050. Parmi ceux-ci, la part des 55 à 64 ans doublera pour s'élever à $24 \%$. Dès 2020, les actifs occupés de 50 à 64 ans constitueront le groupe le plus important de la population active et auront pris le relais des 35-49 ans, aujourd'hui les plus nombreux.

Nul ne sait quel en sera l'effet sur la productivité. Certes, on sait que la capacité de travail physique diminue avec l'âge ; mais l'expérience professionnelle et humaine comme la connaissance en matière d'organisation suffiront-elles à compenser cette diminution dans la société du savoir et une économie tertiarisée? Généralement, les salariés âgés sont moins ouverts aux nouveaux produits et aux procédés innovants, ce qui représente un risque pour l'avenir. D'habitude,
L'Europe perdra influence et compétitivité dans le monde

$7 \%$ d'Européens seulement dans la population mondiale en 2050

Recul de $29 \%$ de la population européenne en âge de travailler...

Et hausse de 8 points du PIB des dépenses de santé et de retraites

Net vieillissement des actifs occupés, notamment en Allemagne

Seront-ils encore productifs et innovants ? 
Seulement si la qualité du capital humain est assurée, ...

... si les transferts sociaux sont réduits au profit de l'investissement ...

... et si l'Europe mise sur le partage mondial du travail

Pourtant, la croissance potentielle de l'UE tombera à 1,25\% en 2050

Des valeurs et comportements peu offensifs... les entreprises hautement spécialisées et dont les activités sont basées sur une forte division du travail confient l'innovation produit et la modernisation des process à leurs jeunes salariés. Les plus âgés sont en charge principalement des produits et services impliquant une approche traditionnelle et depuis longtemps établis sur le marché. C'est donc la plupart du temps grâce à l'embauche de jeunes salariés, frais émoulus du système de formation, que les entreprises renouvellent leur savoir. Faute de jeunes actifs en nombre suffisant, cela ne sera plus possible à l'avenir. En revanche, le déficit physique pourra partiellement être compensé par la mise en œuvre de nouvelles technologies, notamment les TIC.

L'accroissement de la productivité ne pourra s'accélérer que si la population active, dont l'âge augmente alors qu'elle diminue en nombre, bénéficie de la meilleure formation initiale et continue possible. Cela requiert d'importants investissements dans la formation de tous les actifs - des plus jeunes aux plus âgés. Cela exige l'implication des pouvoirs publics et du patronat, mais aussi celle de chaque individu. Car à l'avenir, chaque salarié aura une responsabilité croissante dans sa propre employabilité. La qualité du capital humain sera en effet décisive pour prendre le relais d'un facteur travail sur le déclin.

Par ailleurs, la diminution de la population active devra être compensée également par un recours accru au facteur capital. Autrement dit, il s'agira de mettre à disposition des actifs un parc ultramoderne d'installations et équipements, ainsi que des infrastructures performantes. Pour ce faire, nombre d'Etats membres devront réduire la voilure de leurs systèmes publics de protection sociale, généralement assis sur une généreuse pratique de redistribution et de transferts, et leur adjoindre en compensation des régimes d'assurance par capitalisation. Dans le contexte de ce dramatique vieillissement démographique, les réformes des retraites ne peuvent avoir pour seul objectif de garantir la protection sociale des personnes âgées ; il s'agit bien plus, en réformant la protection vieillesse, de ménager la transition vers une structure productive où un nombre décroissant d'actifs aura à assurer la subsistance d'un nombre croissant de purs consommateurs.

Cela étant, les Européens peuvent aussi mettre à profit l'internationalisation croissante des marchés de biens et de capitaux pour investir massivement des capitaux à l'étranger afin de faire produire hors du marché communautaire les biens qu'ils importeront ensuite pour couvrir leurs besoins. Cela présenterait en outre l'avantage de parer au risque d'une dévalorisation des capitaux placés sur le marché domestique, que ce risque provienne du recul de l'épargne des personnes âgées ou d'une liquidation massive des avoirs par la génération du baby-boom.

Mais cela ne suffira pas à enrayer la perte de dynamisme de l'économie européenne induite par le vieillissement. La Commission européenne a ainsi calculé que, à l'horizon 2040, la croissance potentielle de l'UE, actuellement de $2 \%$ à $2,25 \%$, tombera à environ 1,25\% ; le PIB par habitant perdra en conséquence un cinquième environ de son niveau actuel. Tel sera l'effet des mutations démographiques sur le cadre des activités et, plus encore, sur les valeurs et comportements de la société.

Certes, aucune expérience historique ne permet d'extrapoler l'impact d'une telle modification de la pyramide des âges. On peut néanmoins supposer qu'une population européenne dont un gros tiers aura plus de 60 ans et un dixième plus de 80 ans en 2050 prendra quelque distance avec l'acquisition ultérieure de biens matériels - nettement plus en l'occurrence qu'une population américaine où respectivement une quart seulement et un quatorzième des habitants figureront dans ces classes d'âge. Quels motifs pourraient bien inciter une telle population âgée, confortablement équipée en biens de consommation durable (de l'automobile à l'habitat), à engager des efforts hors du commun pour rivaliser avec d'autres peuples, voire les surclasser? 
Bien sûr, certaines catégories de services, qu'ils soient de type primaire ou qualifié, vont enregistrer une solide croissance; elle sera particulièrement nette dans le domaine de la santé et des soins à la personne. Mais dans le même temps, il est tout aussi vraisemblable qu'une population vieillissante accordera une importance accrue à l'immatériel, notamment à la sécurité, et donc à l'assurance contre le vol ou l'escroquerie des biens acquis tout au long de la vie. L'acceptation du risque diminuera d'autant. Le changement laisse sceptique une population âgée ; rares sont ceux qui ont le courage du renouveau. Or c'est justement l'ouverture à la nouveauté qui est requise pour s'affirmer dans une compétition mondiale avivée. La même attitude est nécessaire pour maîtriser les effets des mutations démographiques.

Proportion des 65 ans et plus par rapport aux 15-64 ans (en \%)

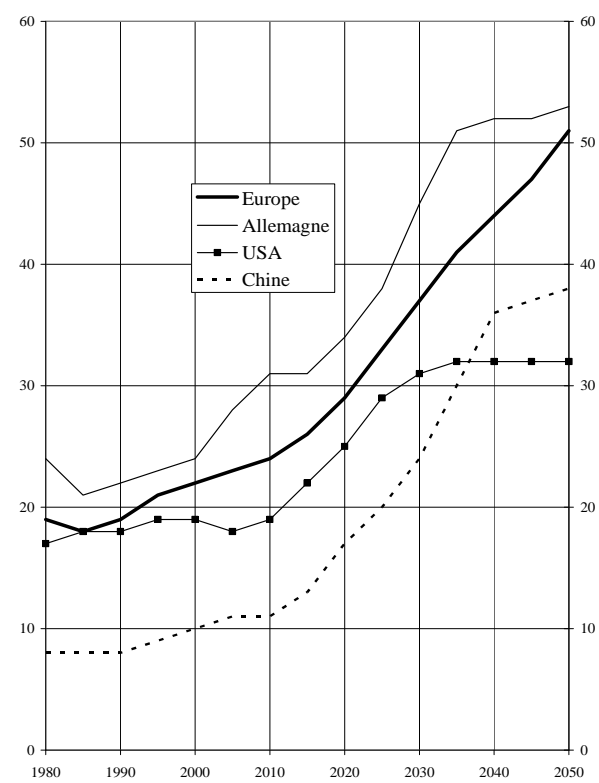

L'accroissement du taux de dépendance

Sources: Population Division of the Department of Economic and Social Affairs of the United Nations Secretariat, World Population Prospects (The 2002 Revision), et World Urbanization Prospects (The 2001 Revision) site : http://esa.un.org/unpp.

Au cours des décennies à venir, l'Europe aura donc à affronter un double défi : le vieillissement de sa population et une concurrence mondiale accrue dont les enjeux sont les ressources et les marchés. Plus elle maîtrisera vite les effets des mutations démographiques en les appréhendant dans leur globalité, mieux elle sera armée dans la compétition mondiale. La priorité est de prendre conscience de l'ampleur de ces mutations et de faire promptement les choix permettant de guider son action. La particularité des mutations démographiques est de s'effectuer insensiblement, de manière rampante, ce qui permet encore à nos responsables du monde politique comme de la société civile, sans oublier les citoyens, de se bercer dans l'illusion en cultivant des certitudes qui, déjà, ont perdu leur fondement. Nos hommes politiques européens sont dès lors appelés à aiguiser la prise de conscience du problème démographique et à développer ainsi la volonté de l'affronter. C'est la condition sine qua non pour que l'Europe préserve sa compétitivité.

En AlLemagne, l'ÉCrasante maJorité de LA POPULATION a pris conscience des mutations démographiques en cours et des problèmes qu'elles soulèvent : près de neuf Allemands sur dix, selon un récent sondage effectué par l'institut lpos. Les deux tiers estiment même que le vieillissement met en danger leur standard de vie. Pourtant, la majorité se refuse à accepter les conséquences qu'il faut en

Un seul remède au déclin : la prise de conscience 
tirer. Ainsi, $80 \%$ des Allemands s'opposent à ce que l'âge légal de la retraite soit relevé à 67 ans. Ils sont tout aussi nombreux à refuser une baisse du niveau des pensions de retraites, une hausse des cotisations sociales ou une réduction des prestations d'assurance maladie. Or dans le même temps, ils sont nombreux à surévaluer les droits qu'ils ont acquis en matière de protection sociale, tout particulièrement en matière de retraites.

La conséquence en est que le gouvernement fédéral se voit confronté à un dilemme. Pour préserver à la fois la compétitivité de l'Allemagne et garantir une protection sociale de haut niveau, il lui faudrait réformer avec plus de conséquence que jusqu'ici le système de protection sociale. Les efforts entrepris dans le domaine de l'assurance maladie avec la Loi de modernisation du système de santé (Gesundheitsmodernisierungsgesetz) ou dans celui de la protection vieillesse avec les Lois sur la soutenabilité de l'assurance retraite (Rentenversicherungsnachhaltigkeitsgesetz) et sur les revenus des personnes âgées (Alterseinkünftegesetz) sont loin d'être suffisants. Mais la coalition fédérale sait parfaitement que si elle poursuit les réformes plus avant, elle ne sera pas reconduite. Elle se contente donc d'une politique de réformes menée comme à contre cœur. Mais il ne sera bientôt plus possible de poursuivre sur la voie de réformes modérées. A moyen terme, l'Allemagne ne pourra plus éluder ni la réforme de fond de son système de protection sociale, ni la révision de son système fiscal, notamment en ce qui concerne le régime des prestations familiales, ni la modernisation de son système de formation, ni l'adaptation de tant d'autres domaines aux conséquences du vieillissement démographique. La priorité, aujourd'hui, est d'en faire prendre conscience les citoyens.

(Traduction : I. Bourgeois)

\section{Indications bibliographiques}

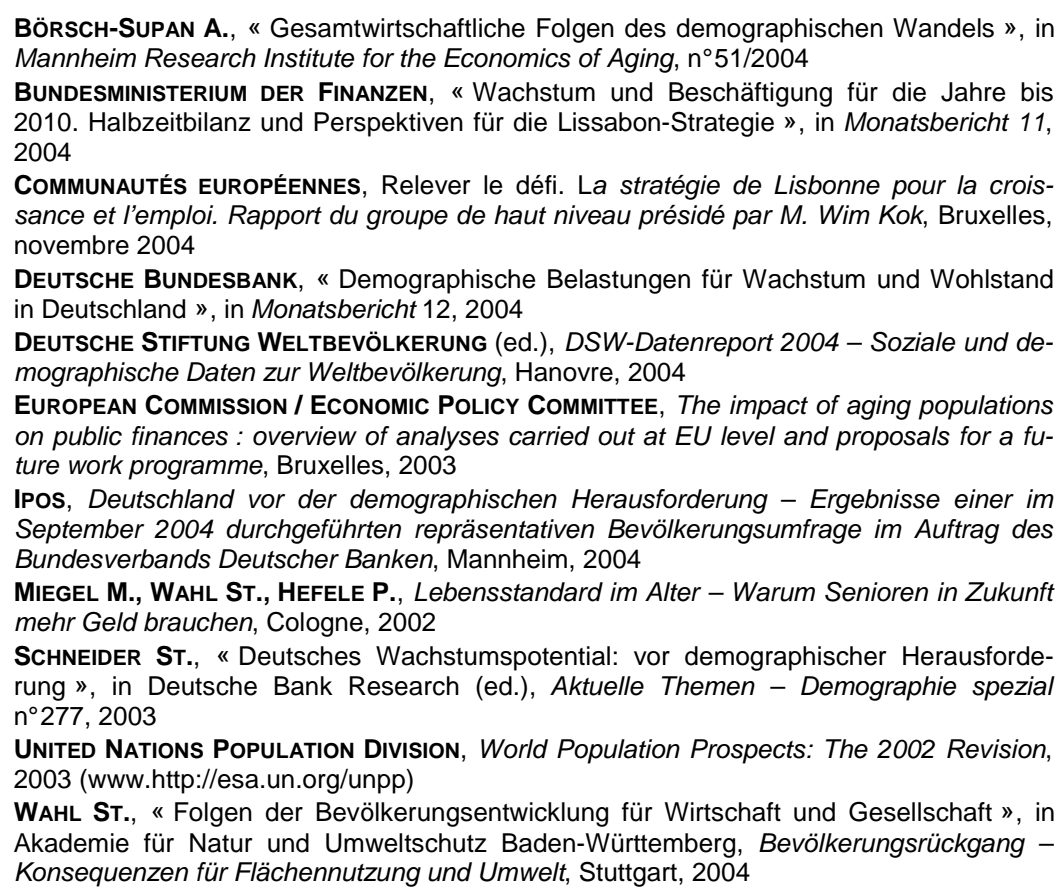

